\title{
Spectrally efficient transmit diversity scheme for differentially modulated multicarrier transmissions
}

\author{
B. Allen, F. Said, G. Bauch, G. Auer and A.H. Aghvami
}

\begin{abstract}
Cyclic delay diversity is a simple, yet effective, transmit diversity scheme for multicarrier based transmissions employing coherent digital linear modulation schemes. It is shown that, for satisfactory operation, the scheme requires additional channel estimation overhead compared to single antenna and traditional space-time coded transmissions owing to the inherent increase in frequency selective fading. The authors analyse the additional channel estimation overhead requirement for a Hiperlan \#2 style system with two transmit antennas operating in a NLOS indoor environment. The analysis shows that an additional overhead of $500 \%$ is required for the candidate system compared to a single antenna system. It is also shown that by employing differential modulation the channel estimation overhead can be eliminated with significant performance improvement compared to a system employing a practical channel estimation scheme. This novel combination, termed 'differentially modulated cyclic delay diversity', is shown to yield a highly spectral efficient, yet simple transmit diversity solution for multi-carrier transmissions.
\end{abstract}

\section{Introduction}

Providing a cost effective wireless architecture with diversity is important when high-performance multimedia services are required. Diversity can be achieved in time, space, frequency or polarisation and can be implemented at the transmitter or receiver or any combination. Recently transmit diversity has received much attention and spectrally efficient solutions have been proposed through the application of space-time codes [1, 2]. One promising transmit diversity technique for multicarrier based transmissions such as orthogonal frequency division multiplexing (OFDM) that employ coherent linear digital modulation schemes is cyclic delay diversity (CDD) $[3,4]$ which has the advantage of having a simple transmit architecture and not requiring any modification to the receiver. Bossert et al. [3] show an OFDM system employing CDD and two transmit antennas to have a bit-error rate performance comparable to the Alamouti scheme described in [2] when a half-rate convolutional channel code is employed. CDD inherently creates additional frequency selective fading compared to single antenna and traditional space-time coded transmissions, which is caused by the cyclic signal delays inserted in each transmit chain (see the CDD block diagram in Fig. 1). The increased frequency selectivity impacts on the channel estimation (CE) requirements and the corresponding pilot signalling overhead of CDD transmissions.

It is well known that differential modulation schemes such as DPSK eliminate the need for channel estimation at the receiver [5]. Thus combining CDD with differential

\section{(C) IEE, 2005}

IEE Proceedings online no. 20040962

doi:10.1049/ip-com:20040962

Paper first received 8th May 2003 and in revised form 13th May 2004

B. Allen, F. Said and A.H. Aghvami are with the Centre for Telecommunications Research, Kings College, University of London, 26-29 Drury Lane, London WC2B 5RL, UK

G. Bauch and G. Auer are with the DoCoMo Euro-Labs, Landaberger Strasse 308-312, Munich 80687, Germany

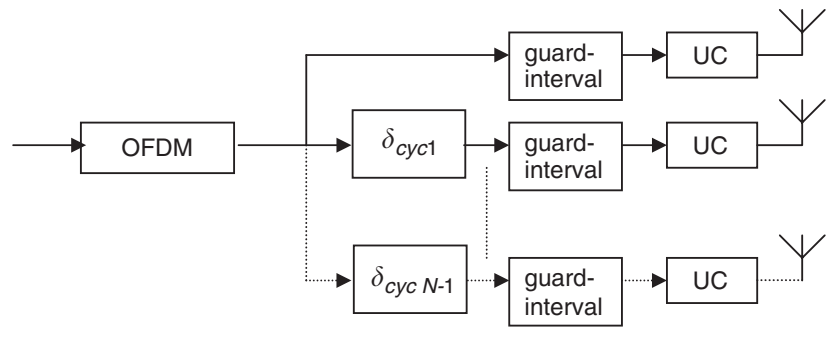

Fig. 1 CCD transmitter architecture

modulation will eliminate channel estimation and will therefore significantly increase the spectral efficiency of CDD transmissions as well as simplifying the transmitter and receiver structures. This novel combination is termed 'differentially modulated cyclic delay diversity' (DMCDD) and is fully described in this paper.

\section{System model}

Figure 1 shows the architecture of a CDD transmitter with $N$ transmit antennas and $N-1$ cyclic delays of length $\delta_{c y c}$ between adjacent antennas. The Figure shows the OFDM symbol is progressively delayed along the array before the guard interval is added, where the delay is a cyclic delay. The operation of the cyclic delay is similar to applying a cyclic guard interval. Thus symbols from the beginning of the OFDM packet are removed and replaced at the beginning, as illustrated in Fig. 2. The Figure shows the OFDM symbols start and stop at the same time on each channel. After the cyclic delay and guard interval are added, the signals are subsequently up-converted (UC) and transmitted. The optimum delay between two adjacent antennas, $\delta_{c y c}=\delta_{c y c}(n-1)-\delta_{c y c}(n)$, has been obtained in [3] for a two-transmit-antenna system and is given by (1).

$$
\delta_{c y c}=\frac{N_{c}}{M} \quad \text { symbols }
$$

Where $N_{c}$ is the number of OFDM subcarriers and $M$ is the cardinality of the $M$-PSK modulation alphabet. Here a 


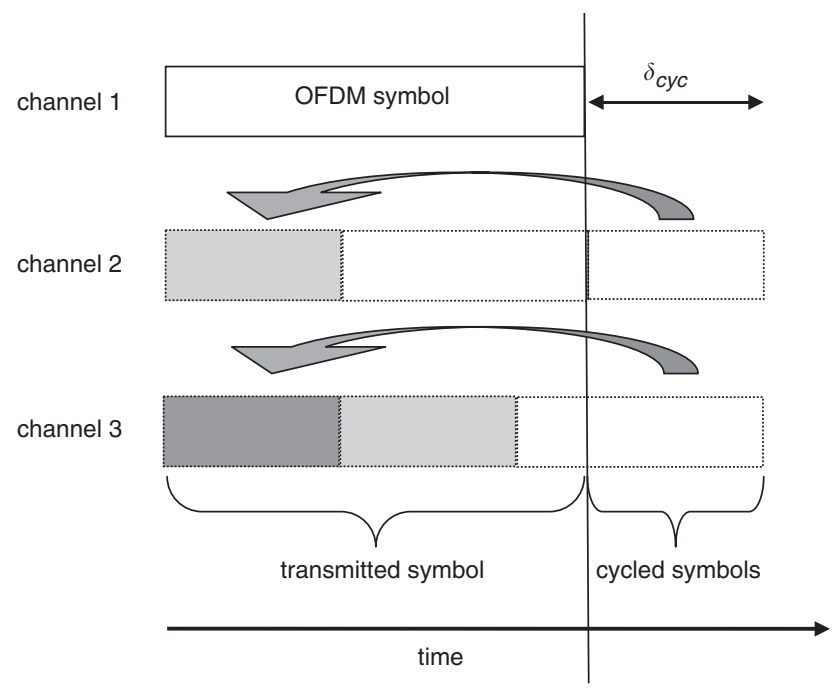

Fig. 2 Cyclic delay signal structure

Hiperlan \#2 style system is considered, hence, $N_{c}=64$ and $M=2$ (i.e. BPSK modulation) is assumed, thus $\delta_{c y c}=32$ samples (or $1.6 \mu \mathrm{s}$ when a data rate of $20 \mathrm{Mbit} / \mathrm{s}$ is assumed). Although $\delta_{c y c}=32$ is deemed to provide the optimum delay, the performance with $\delta_{c y c}=4$ and $\delta_{c y c}=16$ is also investigated and compared to the performance when $\delta_{c y c}=32$. A transmission bandwidth of $20 \mathrm{MHz}$ is assumed here which yields a subcarrier spacing of $F_{c}=312.5 \mathrm{kHz}$. A two-transmit, one-receive-antenna system is considered as this allows easy comparison with the Alamouti scheme in [2] (although CDD can be extended to any number of transmit and receive antennas). The OFDM modem block in Fig. 1 consists of channel coding, interleaver, modulator and IFFT functions. Here, the CDD and DMCDD systems employ a half-rate, memory two convolutional channel code with a constraint length of three and generator polynomials $G(D)=\left(1+D^{2}, 1+D+D^{2}\right)$. The channel code is an integral part of CDD to obtain satisfactory performance and does not detract from the spectral efficiency since most wireless systems specify a channel code as part of the base-line air-interface.

The channel model used for this analysis is shown in Fig. 3 and consists of an impulse response with exponential decay profile, eight independent Rayleigh fading taps and a delay spread of $\tau_{d}=400 \mathrm{~ns}$, which is representative of operation in a NLOS indoor environment. It is also assumed here that the normalised Doppler bandwidth, $F_{d} T_{c}=5 \times 10^{-3}$, and the transmit antennas are mounted sufficiently far apart to yield spatially decorrelated channels in the given environment.

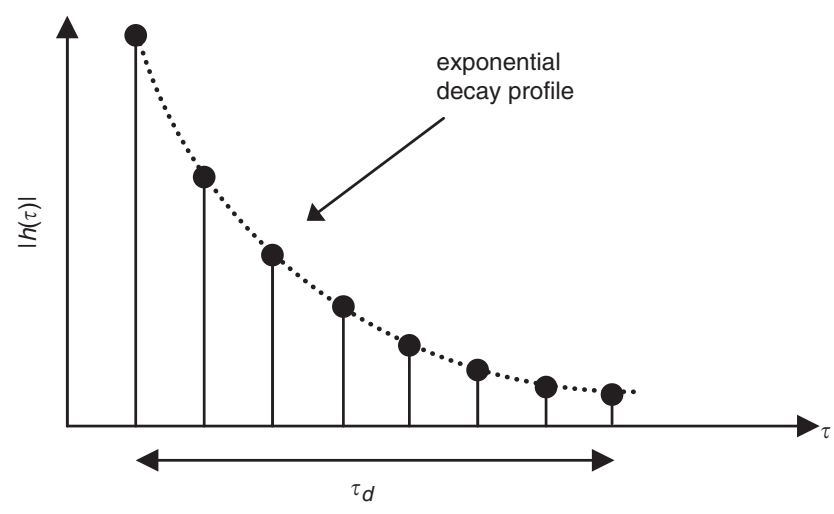

Fig. 3 Channel impulse response model
A standard OFDM receiver architecture is used for CDD reception and consists of: down-converter, guard interval removal; FFT, channel estimator, coherent signal detection, de-interleaver and soft Viterbi decoder. No additional modification is required at the receiver for CDD operation which illustrates a strength of this transmit diversity technique.

\section{Analysis of channel estimation overhead}

For satisfactory $\mathrm{CE}$ in multicarrier systems that employ pilot symbol assisted channel estimation, pilot symbols must be inserted in the frequency domain with a spacing given by (2) when $2 \times$ over-sampling is employed [6]. A twice over-sampling rate for the pilot subcarrier symbols is usually used to suppress channel noise and improve the estimation performance.

$$
\Delta f_{\text {pilots }} \leq \frac{1}{2 \cdot \tau_{d} \cdot F_{c}}
$$

Where $\tau_{d}$ is the channel delay spread and $F_{c}$ is the subcarrier spacing. Thus for the channel model employed here, pilot symbols are inserted in every fourth subcarrier and linear interpolation is performed between pilot symbols to yield satisfactory performance for single antenna operation and when traditional space-time codes such as the Alamouti code are employed. In the later case, pilot symbols are required to be mutually orthogonal between transmit antennas to enable separation of each channel at the receiver. When multiple transmit antennas are employed, the received signal is a vector summation of the signals received from each transmit antenna. In the case of CDD, each received signal is a delayed version of the others, where the delay is a cyclic delay. This process is mapped onto the channel characteristics that correspond to the combined signal observed at the receiver as shown in Fig. 4. The Figure shows the impulse response from the combined channel, where a significant increase in delay spread is observed compared to the signal channel case. Figure 5 shows the corresponding effect in the frequency domain where the instantaneous frequency selective fading is shown for each independent channel and the combined channel. A significant increase in frequency selective fading is shown for the combined signal in the Figure compared to the individual channels, which results from signal combination

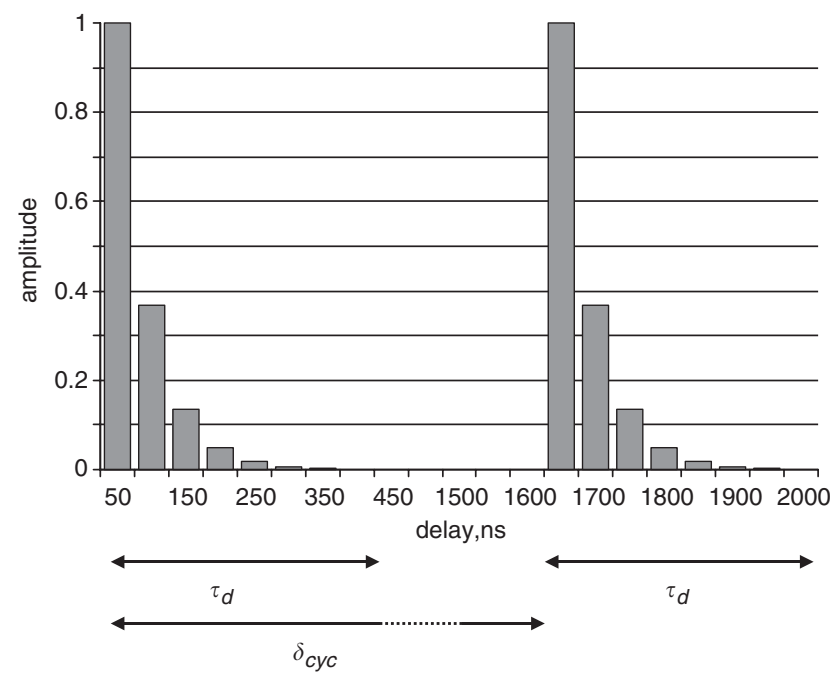

Fig. 4 Combined channel delay characteristics 


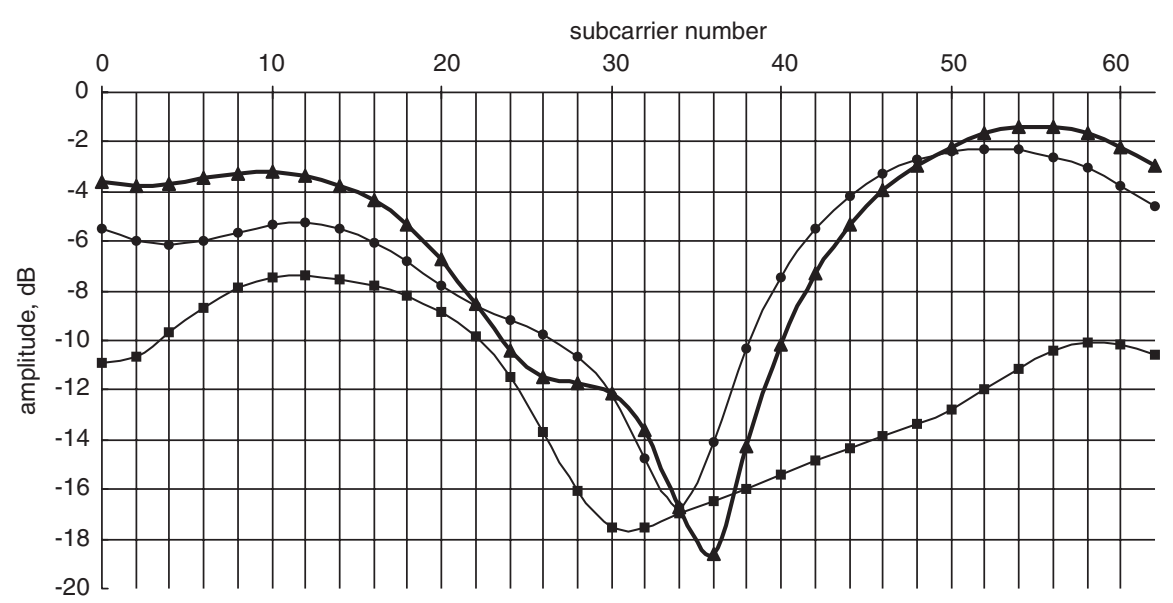

$\longrightarrow$ channel $\longrightarrow$ - channel $2 \multimap$ combined channel

Fig. 5 Example channel frequency responses

of the delayed signals. The combined channel in Fig. 4 shows the delay spread is increased to $\tau_{\text {total }}=2 \mu \mathrm{s}$, where $\tau_{\text {total }}=\tau_{d}+\delta_{\text {cyc }}$. Thus the system requires a pilot signal spacing given by (3).

$$
\Delta f_{\text {pilots }_{C D D}} \leq \frac{1}{2 \cdot F_{c} \cdot\left(\tau_{d}+\delta_{c y c}\right)}
$$

Thus for this example $\Delta f_{\text {pilots } C D D}=0.8$. This is clearly impractical and to obtain optimal operation, the subcarrier spacing and $\delta_{c y c}$ are two parameters that must be appropriately chosen (although this is an unacceptable solution for systems that have already undergone standardisation).

The percentage increase in CE overhead, $\xi$, that results from the additional pilot signals is given by (4). For the system considered here, $\xi=500 \%$.

$$
\xi=\frac{\Delta f_{\text {pilots }}}{\Delta f_{\text {pilots } C D D}} \cdot 100 \%
$$

$\xi$ is expressed in (5) below in terms of $\delta_{c y c}$ and $\tau_{d}$, which is obtained by substituting (2) and (3) into (4).

$$
\xi=\frac{\tau_{d}+\delta_{c y c}}{\tau_{d}} \cdot 100 \%
$$

Although the required pilot signal spacing has been shown to be significantly reduced when CDD is employed, pilot signals are not required to be orthogonal between transmit antennas as required by Alamouti and other space-time codes [7]. This property is not required since the individual channels are not estimated and consequently simplifies the transmitter and receiver complexity.

\section{Differentially modulated cyclic delay diversity}

The previous Section has shown that the CE overhead required for CDD is significantly more than that of systems employing a single antenna or traditional space-time codes where the composite channel is not estimated. However, the relative simplicity of CDD and the performance potential makes CDD an attractive transmit diversity solution for multicarrier systems if a suitable solution for reducing or eliminating the associated CE overhead is obtained. To this end, differentially modulated cyclic delay diversity (DMCDD) is proposed here.
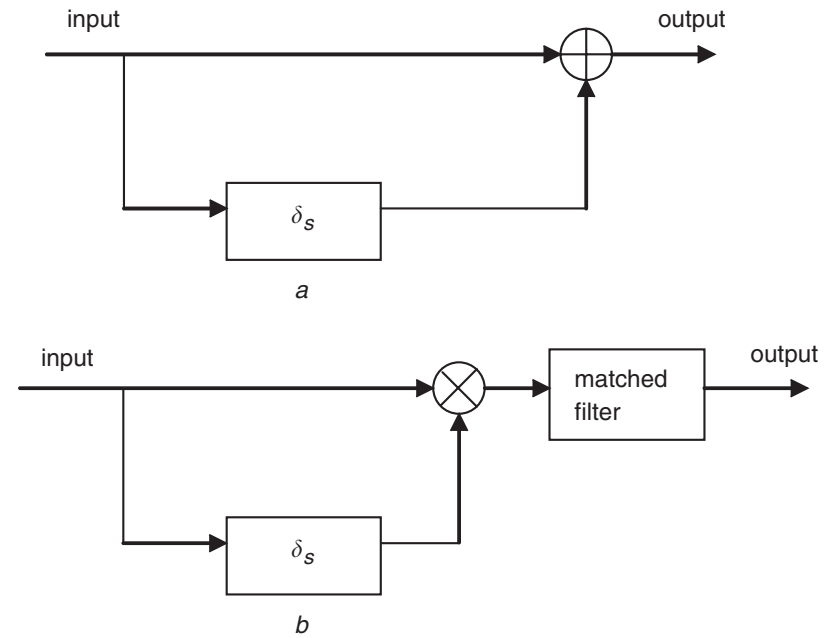

Fig. 6 Differential modulation $a$ Encoding process $b$ Decoding process

DMCDD combines differential modulation, such as DPSK [5], with CDD. DPSK employs differential encoding of the signal at the transmitter and non-coherent detection at the receiver, as shown in Fig. 6, where $\delta_{s}$ is a one-symbol delay. The output from the encoder is the difference between two adjacent input symbols, thus the transmitted symbol, $c(k)$, is the phase difference between the previously encoded symbol and the $k^{\text {th }}$ message, $m(k)$, as given by (6).

$$
c(k)=c(k-1) \oplus m(k)
$$

Where $\oplus$ is the modular- 2 addition of the symbols.

The detection process is similar, where two adjacent (differentially encoded) symbols are multiplied and the combined signal is then detected via a matched filter and decision stage as shown in Fig. 6b. Thus, the detector does not require a channel estimate to be computed since the difference between two received signals provides the required reference signal.

There is, however, a performance penalty associated with differential modulation since the reference signal used in the detection process is obtained from two noisy received symbols, therefore increasing the required signal-to-noise ratio for a given bit-error probability by approximately $3 \mathrm{~dB}$ compared to coherent detection. Although a performance 
penalty is incured, there are clear advantages of employing differential modulation, namely:

- pilot symbol overhead is eliminated (a one-off reference symbol is transmitted instead);

- Channel estimation is not required at the receiver; and

- Interpolation is not required at the receiver.

Thus, the transmitter and receiver are significantly simplified and the spectral efficiency is increased, especially in the case of CDD where CE overhead is necessarily very high as shown in Section 3. Furthermore, transmit diversity techniques employing coherent detection often require the transmitter power to be increased during pilot symbol bursts since these symbols do not incur the added protection from diversity that data symbols have. This requirement is eliminated when differential modulation is employed.

Figure 7 shows the block diagram of a DMCDD system. The key difference between this system and that of a coherent CDD system is the differential encoding at the transmitter that is required and the elimination of pilot signals. At the receiver, differential detection is employed but channel estimation and interpolation are not required.

It has already been stated that differential detection requires two received symbols to be processed, where the difference between them is used to obtain the transmitted message symbol. This requires the channel phase characteristics to be almost stationary between the two signals to obtain high performance. In the case of multicarrier transmissions, differential modulation can take place in the frequency domain (between adjacent subcarriers) or in the time domain (between adjacent OFDM symbols). Here, it has been applied in the time domain as this avoids large channel variations caused by the severe frequency selective fading that results from CDD, as described in Section 3. Note, however, that as the normalised Doppler bandwidth increases, the signal variation between adjacent OFDM symbols will also increase.

\section{Simulation parameters and results}

The performance of CDD with two configurations of pilot symbol spacing and DMCDD has been ascertained by Monte-Carlo simulation for the system configuration and operating environment described in Section 2 and the results are presented in this Section.
Section 3 presented an analysis of the CE overhead required for $\mathrm{CDD}$ and showed that pilot symbols are required in every fourth subcarrier for single antenna operation and operation with traditional space-time codes, and when CDD is employed pilot symbols are required in every 0.8 subcarriers. This is clearly impractical and would require fundamental changes to the system parameters for optimum performance.

Figure 8 shows the simulated bit-error rate (BER) performance as a function of energy-per-bit to noise power spectral density $\left(E_{b} / N_{o}\right)$ for a reference single transmit antenna system with perfect $\mathrm{CE}$ (pilots inserted in every subcarrier) and with pilot symbols inserted in every fourth subcarrier. Interpolation is performed between subcarriers without pilot symbols by using a linear interpolation scheme, the pilot symbols are updated every eighth OFDM symbol in the time domain assuming a normalised Doppler bandwidth of $F d T c=5 \times 10^{-3}[6]$ and results have been accumulated over 1000 errors per simulation point. These reference curves allow performance to be compared with the curves that are shown for the above parameters when two transmit antenna are employed and $\delta_{c y c}=4,8$ and 32 for the case with perfect $\mathrm{CE}$ and with practical $\mathrm{CE}$.

The results show that, when two transmit antennas are employed, an additional diversity gain from the system compared to single antenna operation is observed. The diversity gain is shown to increase as $\delta_{c y c}$ is increased and a diversity gain of $4.5 \mathrm{~dB}$ is achieved when $\delta_{c y c}=32$ and for $\mathrm{BER}=10^{-3}$. An error floor is shown to exist for all values of $\delta_{c y c}$ when practical CE is employed, with the error floor becoming worse as $\delta_{c y c}$ increases. This is due to the CE not being able to accurately estimate the channel when additional frequency selectivity is introduced by the cyclic delay.

Figure 9 shows the BER performance of DMCDD compared to $\mathrm{CDD}$ with pilot symbols inserted in every fourth subcarrier. A reference curve for single antenna operation with differential modulation is also shown which allows a comparison with two antenna operation to be observed. Curves are shown for $\delta_{c y c}=4,8$ and 32. It is clear from the Figure that DMCDD performance does not exhibit the error floor observed with CDD since it is not affected by the increased channel frequency selectivity. Performance of DMCDD is also shown to improve as $\delta_{c y c}$ is increased, with the best performance obtained when $\delta_{c y c}=32$. A further advantage of DMCDD is the increased
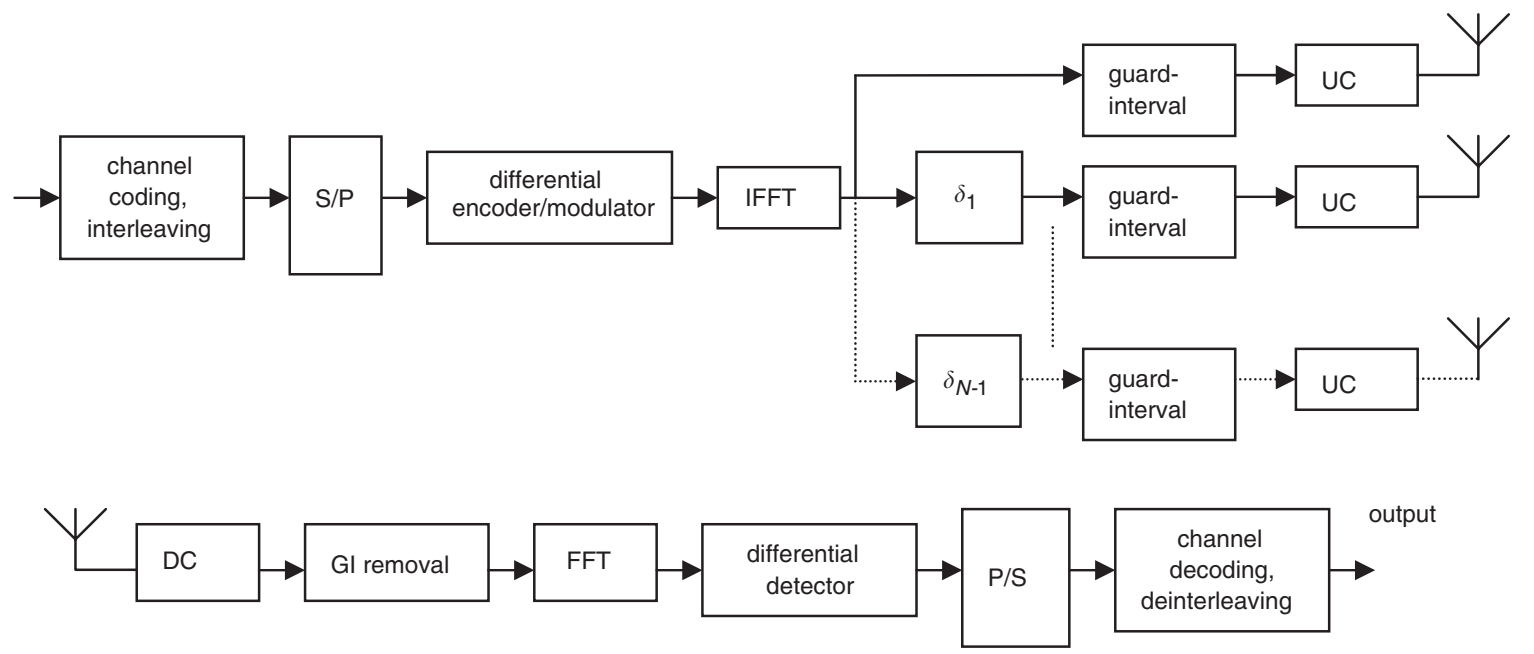

Fig. 7 Block diagram of a DMCDD system 


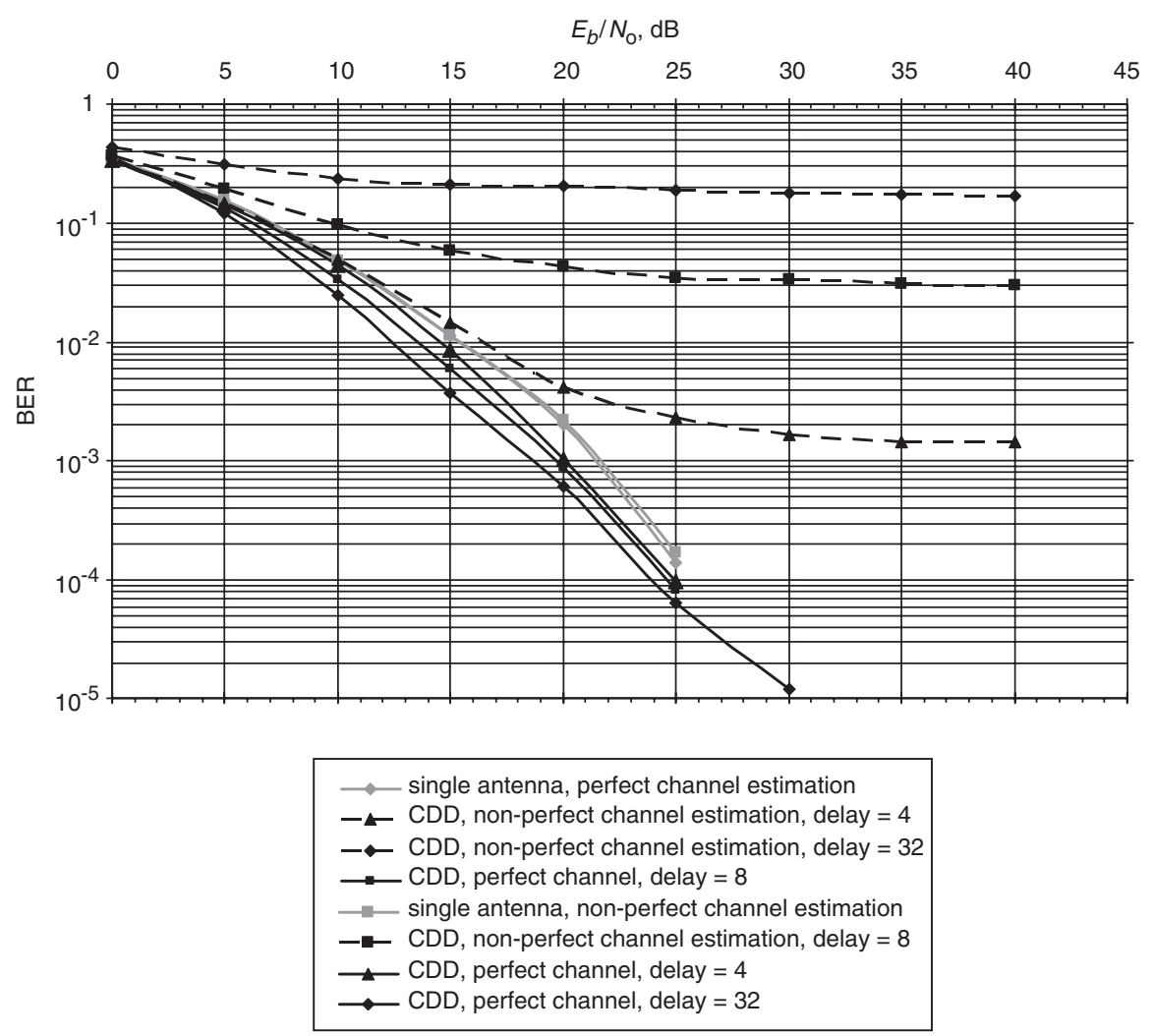

Fig. 8 Performance of CDD at a normalised Doppler bandwidth of $F_{d} T_{c}=5 \times 10^{-3}$ with $\delta_{c y c}=4,8,32$ and with perfect and practical CE Reference curves for one antenna operation are also shown

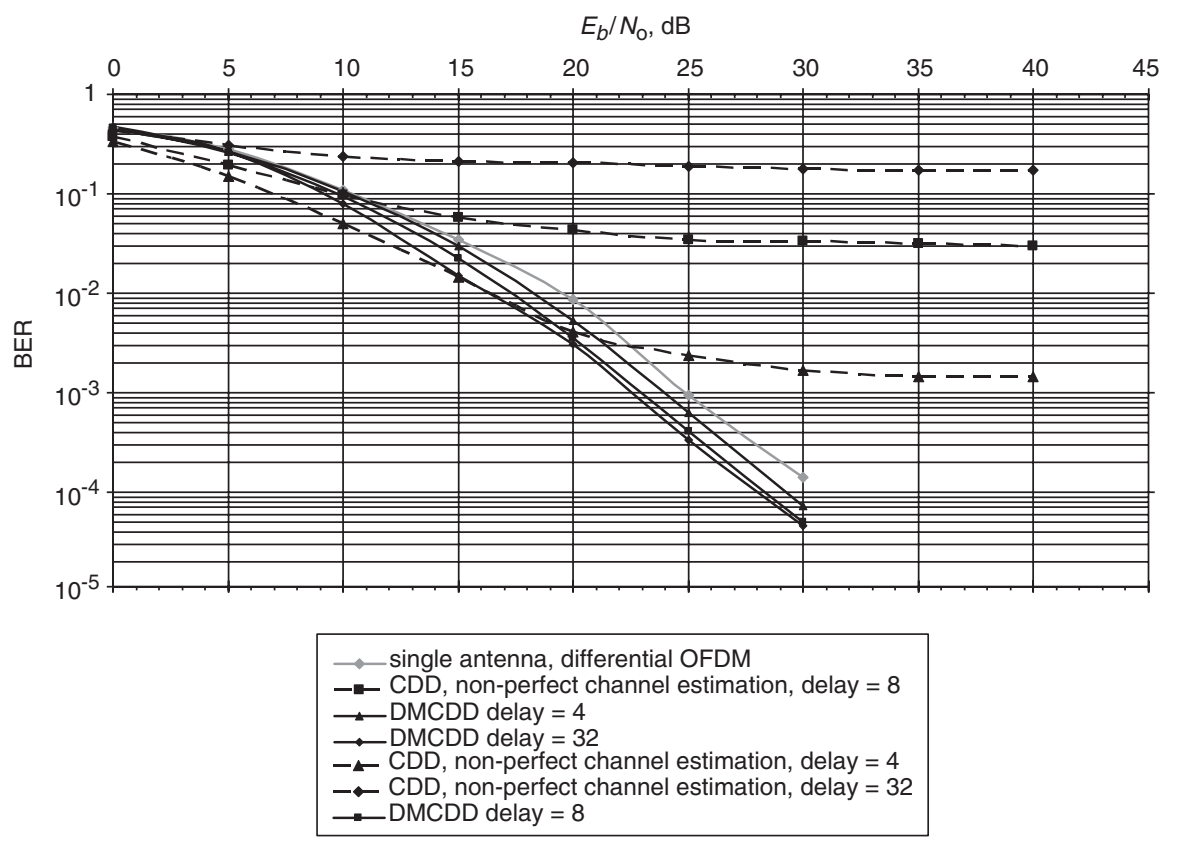

Fig. 9 Performance of $C D D$ with practical $C E$ and DMCDD at a normalised Doppler bandwidth of $F_{d} T_{c}=5 \times 10^{-3}$ with $\delta_{c y c}=4,8$ and 32 Reference curve for one antenna operation is also shown

spectral efficiency that is obtained by omitting the pilot symbols, which in this example pilot signals are inserted in every fourth subcarrier and yields a $25 \%$ increase.

Figure 10 shows the BER performance of DMCDD for various normalised Doppler bandwidths $\left(F_{d} T_{c}\right)$. It is clear from the Figure that increases in $F_{d} T_{c}$ destroys the required correlation between the adjacent OFDM symbols which is essential for the DMCDD operation. This is evident by the appearance of the error floor for $F_{d} T_{c}=0.1$. A solution to this problem can be found by applying the iterative decoding with the decision-feedback differential modulation suggested in [8].

\section{Conclusions}

This paper has shown that CDD increases the delay spread and the associated frequency selective fading of the combined received signal due to the additional cyclic delay 


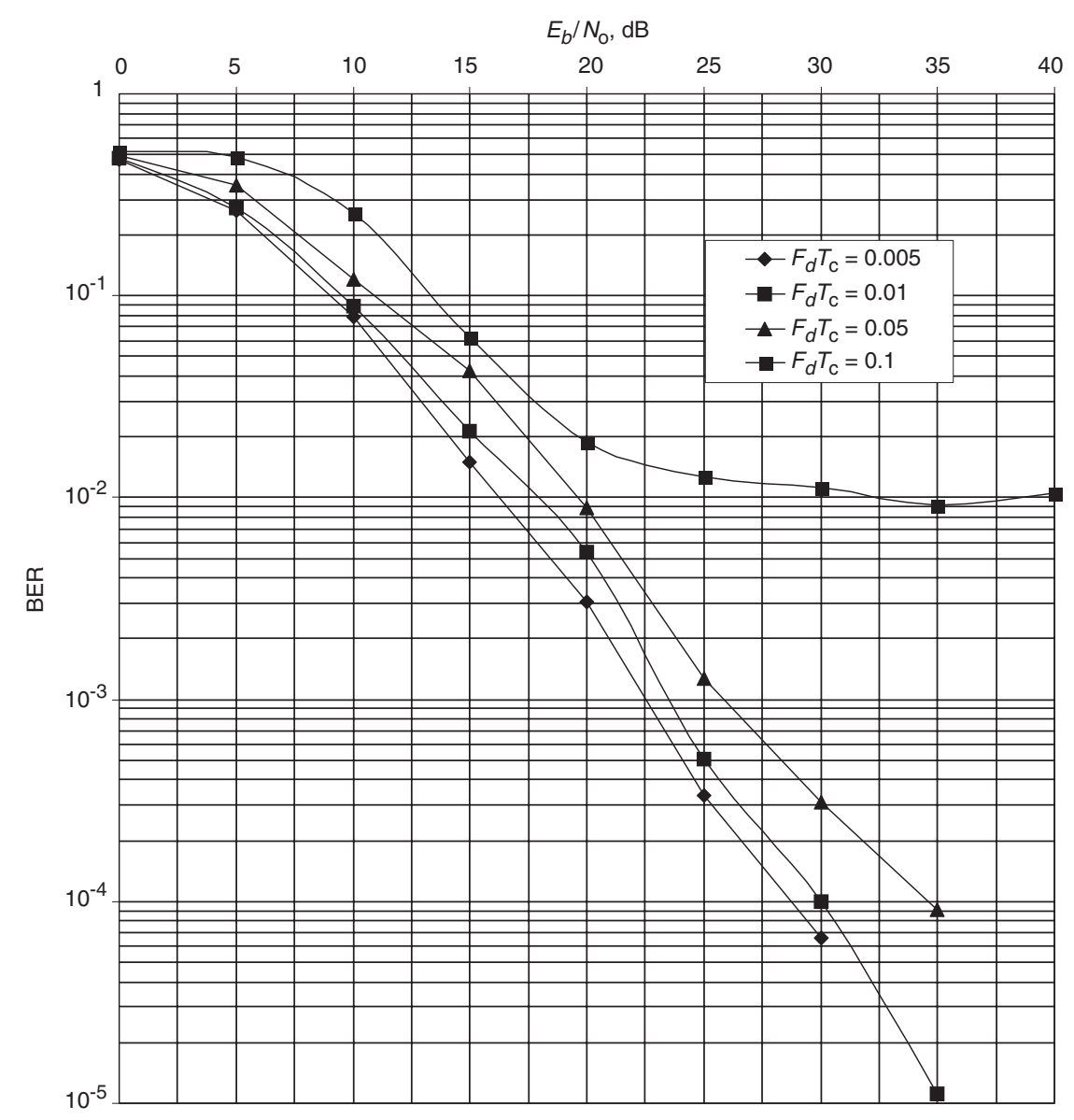

Fig. 10 Performance of DMCDD of normalised Doppler bandwidths

inserted in each transmit path. This improves the system performance due to the added diversity but requires an increased channel estimation overhead. Furthermore, the frequency offset required between pilot signals for optimal performance is shown to be less than the subcarrier spacing for the candidate Hiperlan \#2 style system when CDD is employed, therefore requiring the spacing and transmit delay to be appropriately chosen or suboptimal performance to be obtained. The results show the channel estimation overhead of the candidate system to be increased by $500 \%$.

As a solution to the significant $\mathrm{CE}$ overhead required for CDD, DMCDD had been proposed. This novel transmit diversity technique combines CDD with differential modulation, therefore eliminating the requirement of pilot symbols. The simulated performance of the Hiperlan \#2 style system employing this new transmit diversity technique with two transmit antennas and operating in an indoor NLOS environment shows that the error floor, which occurs when a practical CE scheme is employed, has been eliminated. Thus DMCDD demonstrates a highly spectral efficient transmit diversity technique that is very simple and effective and retains the advantage of not requiring pilot symbols and eliminates the requirement of channel estimation and interpolation at the receiver.

\section{Acknowledgments}

Kings College, London gratefully acknowledges the research fellowship provided by DoCoMo Euro-Labs, Munich, Germany.

\section{References}

1 Tarokh, V., Seshadri, N., and Calderbank, A.: 'Space-time codes for high data rate wireless communications: performance criterion and code construction', IEEE Trans. Inf. Theory, 1998, 44, (2)

2 Alamouti, S.: 'A' simple transmit diversity technique for wireless communications', IEEE J. Sel. Areas Commun., 1998, 16, (8), pp. $1451-1457$

3 Bossert, M., Huebner, A., Schuehlein, F., Haas, H., and Costa, E.: 'On cyclic delay diversity in OFDM based transmission schemes'. Presented at 7th Int. OFDM Workshop, Hamburg, Germany, September 2002

4 Dammann, A., and Kaiser, S. 'Low complexity standard conformable antenna diversity techniques for OFDM systems and its application to the DVB-T system,'. Presented at 4th Int. ITG Conf. Source and Channel Coding, Berlin, Germany, 2002

5 Wilson, S.G.: 'Digital modulation and coding' (Prentice Hall, 1996), pp. $216-220$

6 Yang, B., Cao, Z., and Letaief, B.: 'Analysis of low-complexity windowed DFT-based MMSE channel estimator for OFDM systems', IEEE Trans. Commun., 2001, 49, (11)

7 Naguib, A., Tarokh, V., Seshadri, N., and Calderbank, R.: 'A spacetime coding modem for high-data rate wireless communications', IEEE J. Sel. Areas Commun., 1998, 16, (8), pp. 1459-1478

8 Lampe, L., and Schober, R.: 'Iterative decision feedback differential demodulation of bit-interleaved MDPSK for flat Rayleigh fading channels', IEEE Trans. Commun., 2001, 49, pp. 1176-1184 